\title{
A Novel Repair Technique for the Internal Thermal Control System Dual-Membrane Gas Trap
}

\author{
Thomas O. Leimkuehler and Vipul Patel \\ Honeywell, Inc. \\ Daniel R. Reeves \\ The Boeing Company \\ James M. Holt \\ NASA Marshall Space Flight Center
}

Copyright @ 2005 SAE International

\begin{abstract}
A dual-membrane gas trap is currently used to remove gas bubbles from the Internal Thermal Control System (ITCS) coolant on board the International Space Station (ISS). The gas trap consists of concentric tube membrane pairs, comprised of outer hydrophilic tubes and inner hydrophobic fibers. Liquid coolant passes through the outer hydrophilic membrane, which traps the gas bubbles. The inner hydrophobic fiber allows the trapped gas bubbles to pass through and vent to the ambient atmosphere in the cabin. The gas trap was designed to last for the entire lifetime of the ISS, and therefore was not designed to be repaired. However, repair of these gas traps is now a necessity due to contamination from the on-orbit ITCS fluid and other sources on the ground as well as a limited supply of flight gas traps. This paper describes a novel repair technique that has been developed that will allow the refurbishment of contaminated gas traps and their return to flight use.
\end{abstract}

\section{INTRODUCTION}

The Internal Thermal Control System (ITCS) was developed [1] to provide cooling for various life support equipment, avionics, and experiment racks on the International Space Station (ISS). The dual loop system is comprised of a low temperature loop (LTL) and a moderate temperature loop (MTL). A gas trap [2-4] is utilized on the Pump Package Assembly (PPA) to remove non-condensed gases from the ITCS coolant in order to prevent depriming, overspeed, and shutdown of the centrifugal pump and maintain pump performance. The gas trap provides the capability to vent the air bubbles that are ingested into the ITCS during routine maintainance and replacement of ITCS system components.
The current ITCS gas trap uses 84 concentric membrane tube pairs that are formed into a cylindrical membrane module and then encased within a titanium housing and cover, as shown in Figure 1. During operation, as shown schematically in Figure 2, the ITCS coolant flows into the annular space between the two membranes. The much smaller inner tube, or hollow fiber, is composed of microporous, hydrophobic polypropylene (PP) material. The larger outer tube is composed of porous, hydrophilic Nylon-11 material. The Nylon-11 hydrophilic outer tube allows water to pass through it while trapping any gas bubbles that are present in the coolant. The trapped gas is forced to flow out of the system to ambient through the PP hydrophobic inner fiber and then through a vent tube in the gas trap housing cover.

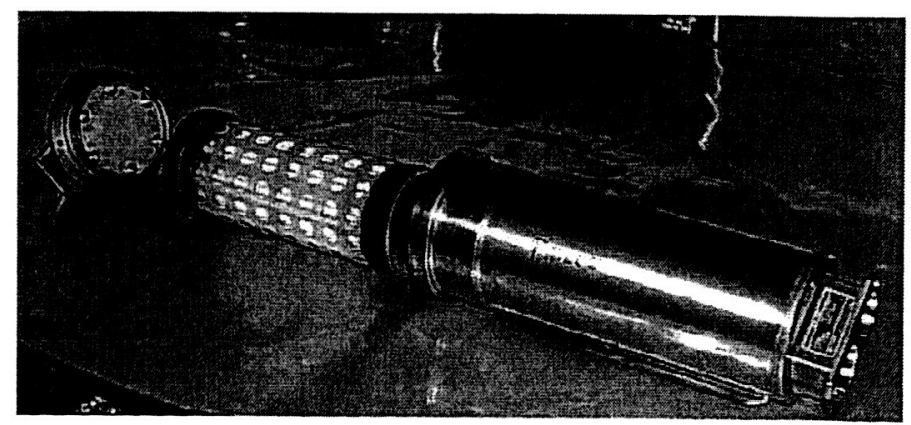

Figure 1. Gas trap subassemblies (I-r: cover, membrane module, housing). 


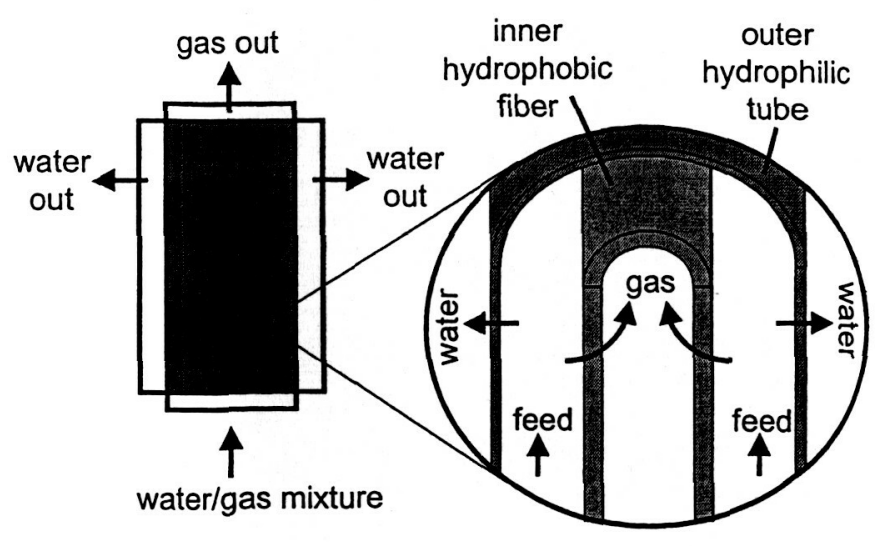

Figure 2. Operation of dual-membrane gas trap

Previous papers [5-6] have discussed fouling of gas traps from nickel contamination in the on-orbit ITCS coolant, and the subsequent cleaning and successful refurbishment of these gas traps. In these cases, nickel contamination fouled the hydrophilic membranes, which resulted in an elevated pressure drop of the coolant flowing through the gas trap. This nickel contamination has been successfully cleaned with a dilute hydrochloric acid solution.

Recently, however, several gas traps have been contaminated on the ground such that the hydrophobic fibers have been contaminated, blocking the venting of gas through these porous hollow fibers. The identity of the contamination in these ground cases includes Braycote grease and microbial growth, both of which have been difficult to remove by cleaning methods that would not cause damage to the hydrophilic tubes. Therefore a new approach was needed to address this challenge.

\section{METHODS INVESTIGATED}

Since efforts to clean some types of contamination from the hydrophobic fibers have been unsuccessful, an alternative approach was needed. Rather than try to clean the contamination from the fibers, it was proposed to remove and replace the fibers in the membrane module. Two methods for replacing the hydrophobic fibers were developed to determine which would be the most effective. Both involved drilling out the hydrophobic fiber. The first of the two methods entails the installation of the fiber by itself using epoxy (Figure 3 ). The second method, installation of the fiber and a portion of the plug (Figure 4), requires additional drilling with a larger bit to create a countersink for the plug to be attached in with the adhesive. The plan was to replace a portion of the hydrophobic fibers using the fiber-only method and the remaining portion using the fiber-plug method.

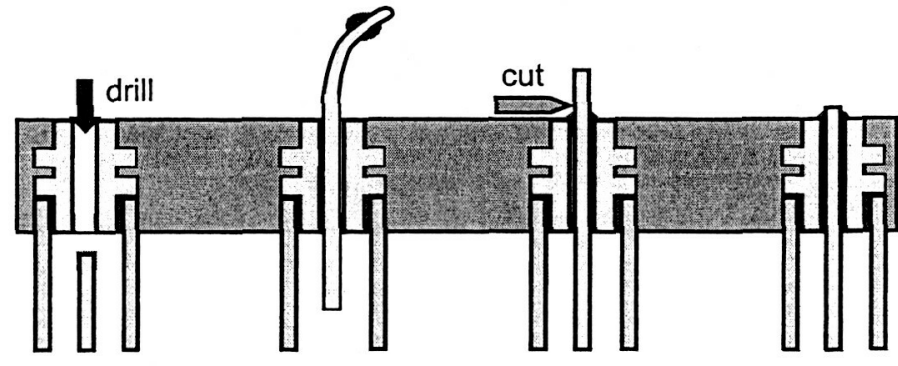

Figure 3. Fiber-only replacement method.

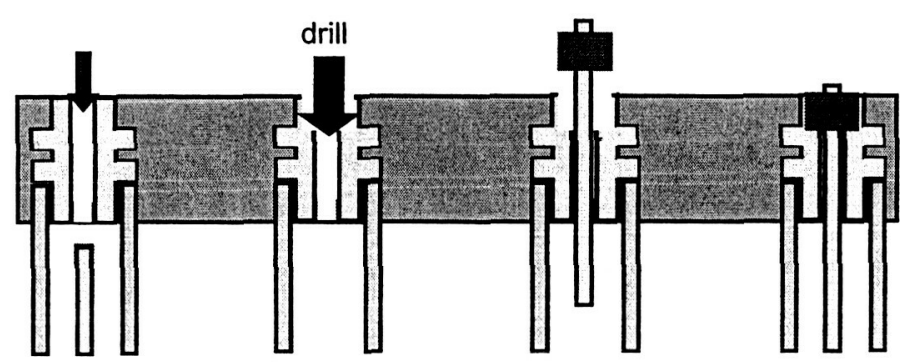

Figure 4. Fiber-plug replacement method.

\section{REPAIR PROCESS}

The research membrane module was initially subjected to selected portions of the acceptance test sequence to establish a baseline for comparison between pre-repair and post-repair results. The hydrophobic fibers were drilled out of the module and replaced via the two installation methods with new hydrophobic fibers.

\section{PRE-REPAIR MODULE EVALUATION}

Before beginning the repair on the research module, the feasibility of the two repair methods was evaluated. The goal of this initial evaluation was to verify that the slightly modified configurations of repaired hydrophobic fibers could withstand operating pressures. More specifically, the test was to check the integrity of the adhesive, used to attach the plug and fiber, at high pressures. Ten holes were drilled into a sheet of Plexiglass and five fiber-plug assemblies and five fiber-only assemblies were installed. Water, pressurized to a pressure of 100 psig was applied to the side of the Plexiglass with the hydrophobic fiber. No damage or leakage resulted from this test, verifying the viability of the repair method. 
New hydrophobic fibers were then prepared for installation into the membrane module. The fibers were cut to length, cleaned, dried, and subjected to screening tests. After the screening tests were completed, 60 fibers were selected for use with the fiber-only replacement method and 20 fibers were selected for the fiber-plug method. (Four of the 84 tube pairs in the research membrane module were removed, leaving the remaining 80 tube pairs for replacement of their hydrophobic fibers.)

\section{PREPARATION OF THE RESEARCH MEMBRANE MODULE}

Next the research module was prepared for fiber replacement. The 80 old hydrophobic fibers were drilled out of the module. After drilling, a number of the old hydrophobic fibers were easily removed from the module as shown in Figure 5. Shaking the membrane module was necessary to remove some of the debris produced by the drilling. Next, 20 of the holes, evenly distributed across the surface of the membrane module, had the additional countersinks drilled for the 20 fiber-plug installations. Figure 6 shows both the drilled and the countersunk holes drilled into the module header. Blowing nitrogen gas through each tube in the membrane module removed some of the debris as well as flushing with deionized water. About seven or eight old hydrophobic fibers were not removed and remained inside their respective hydrophilic tubes, but this should not impede the performance of the gas trap.

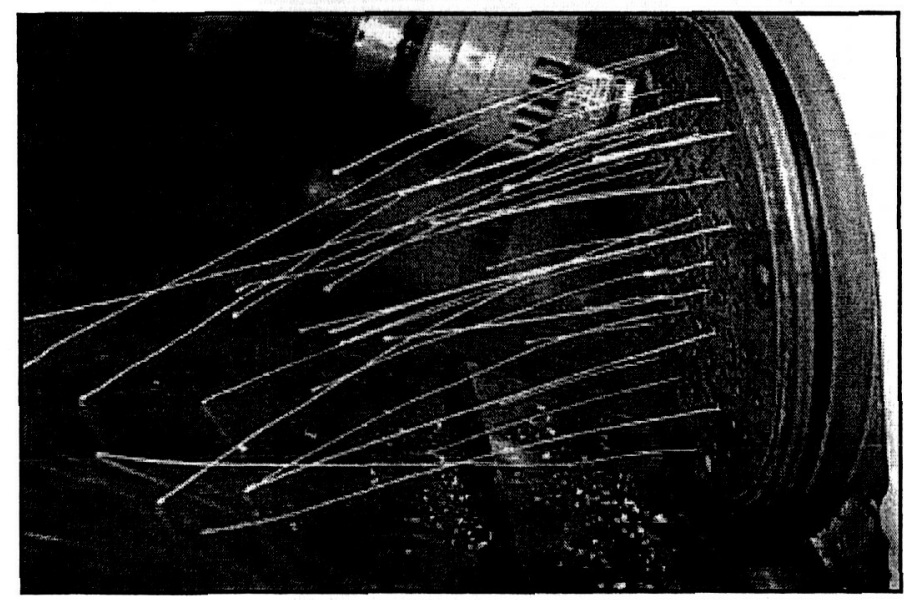

Figure 5. Removal of old fibers from the membrane module.

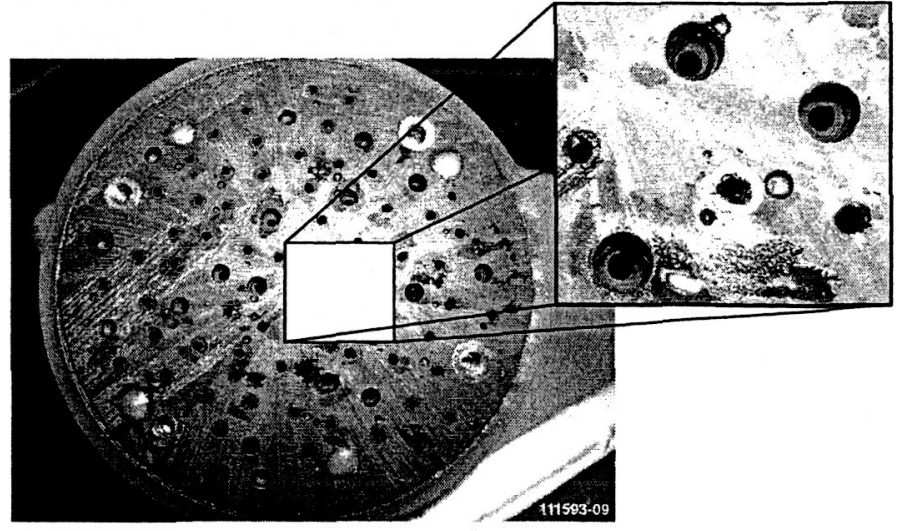

Figure 6. Membrane module gas-side header showing both drilled and countersunk holes.

\section{INSTALLATION OF FIBERS}

Installation of the 60 fibers using the fiber-only method was completed first. Insertion of the fibers was somewhat difficult depending on how the fiber was inserted and the curvatures of the fibers. One of the hydrophobic fiber installations was plugged unintentionally with adhesive. If this had been a flight module, the fiber could have been drilled out and replaced again.

Once installation using the fiber-only method was complete, the short fiber-plug assemblies were cut such that only a small portion of the plug remained for installation into the countersink that had been drilled into the membrane module header. The installation of the fibers with the plugs proved to be challenging because the fiber tended to fold from the weight of the plug. Additionally, the final insertion of the plug portion was a blind operation, allowing for the possibility of damage to the fiber during insertion. Adhesive was used to attach the plugs into the module. Figure 7 shows a side view of the gas-side header on the repaired module before the fibers were trimmed.

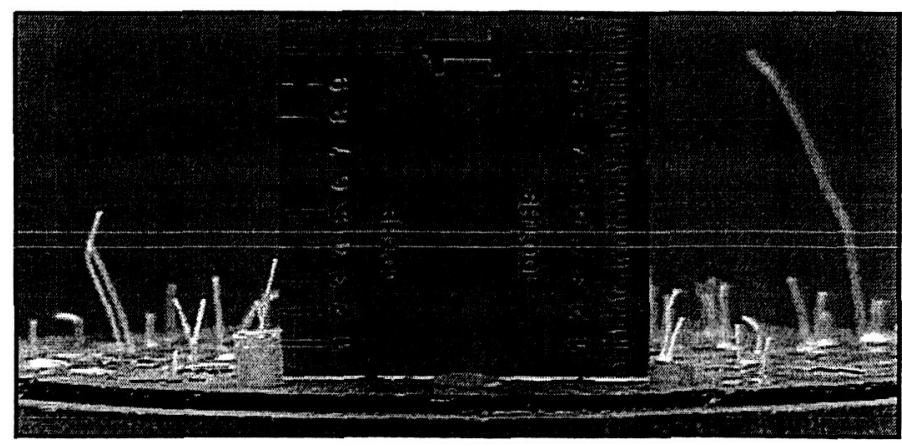

Figure 7. Side view of repaired module gas-side header showing fibers replaced with fiber-only and fiber-plug methods before being trimmed. 
The portion of the fibers still sticking out of the module from both installation methods was cut off and the entire module was put into an oven for curing the epoxy. The module was then cleaned and dried and ready for postrepair evaluation.

After installation, the fiber-only method became the preferred candidate for a replacement method. The fiber-only installation is much simpler when compared to the fiber-plug installation. The fiber-plug installation required the additional steps of drilling the countersink and cutting the plug. Insertion of the fibers was also more difficult with the fiber-plug method due to the weight of the plug still attached. Inserting the plug, using the fiber-plug method, into the membrane module header was a blind operation that could result in damage to the hydrophobic fiber. The fiber-only installation is easier and requires less assembly time.

\section{POST-REPAIR MODULE EVALUATION}

During the initial post-repair evaluation tests, five of the tube pairs in the research module having ruptured tubes were identified and plugged. It was known before this research module was used to investigate this repair technique that the module had ruptured tubes; the repair procedure did not cause the tubes to be ruptured. Also, one additional hydrophobic fiber was observed to be leaking water to the gas side header. This was plugged with epoxy before subjecting the module to proof pressure, water leakage, and the final gas removal test. Note that if this had been a flight module, the leaky fiber could have been drilled out and replaced again. The final configuration of the repaired research module therefore had a total of 10 plugged tubes (4 removed, 5 ruptured hydrophilic tubes, 1 leaking hydrophobic fiber), which is within the allowed limit of 15 plugged tubes. The results of the evaluation tests are presented in the next section.

\section{RESULTS}

The significant changes in performance of the research membrane module from before the repair versus after the repair are presented in Table 1. Prior to the repair process, the gas trap removed less than $10 \%$ of the injected gas. After repairing the module, performance increased to $100 \%$ gas removal.

The $\Delta \mathrm{P}$ of the gas trap after repair, $3.79 \mathrm{psid}$, was slightly higher than the pre-repair $\Delta \mathrm{P}$ of 3.52 psid. This is expected since the ruptured tubes that were plugged relieved pressure within the gas trap. By plugging the tubes, the resistance to flow of the gas trap increased, therefore slightly increasing the overall $\Delta P$. The final $\Delta P$ and bubble removal performance numbers are in family and consistent with that of new gas traps.
Table 1. Comparison of membrane module pre-repair and post-repair evaluation results.

\begin{tabular}{|l|c|c|c|}
\hline Parameter & $\begin{array}{c}\text { Pre-Repair } \\
\text { (4 Tubes } \\
\text { Plugged) }\end{array}$ & $\begin{array}{c}\text { Post-Repair } \\
\text { (10 Tubes } \\
\text { Plugged) }\end{array}$ & $\begin{array}{c}\text { Requirement } \\
\text { \{Note 1\} }\end{array}$ \\
\hline Collection Time (min) & 2 & 5 & 5 \\
\hline $\begin{array}{l}\text { Volume of Gas Injected } \\
\left(\mathrm{cm}^{3} \text { at 1 atm) }\right.\end{array}$ & 2086 & 5215 & N/A \\
\hline $\begin{array}{l}\text { Gas Bypassed } \text { (Note 2\} } \\
\left(\mathrm{cm}^{3} \text { at 1 atm) }\right.\end{array}$ & 1900 & 0 & $<685$ \\
\hline $\begin{array}{l}\text { Gas Trap } \Delta P \text { during } \\
\text { Gas Removal (psid) }\end{array}$ & 7.76 & 4.76 & N/A \\
\hline $\begin{array}{l}\text { Gas Trap } \Delta P \text { during } \\
\text { Flow Rate Test (psid) }\end{array}$ & 3.52 & 3.79 & $<5$ \\
\hline
\end{tabular}

Note 1: Up to 15 tubes are allowed to be plugged in flight units

Note 2: This represents the volume of gas that was not trapped by the gas trap

\section{CONCLUSION}

Based on the results from the repair process, the repair of the research gas trap membrane module was a success. Fiber-only replacement of the hydrophobic fibers is the best method for repair because of the ease of installation. After repair, all of the gas introduced into the system during the gas removal performance test was removed and there was no water leakage from the gas side header. Additionally, a membrane module can be repaired repeatedly using this repair technique. The success of this repair has provided a viable method of membrane module refurbishment.

\section{REFERENCES}

1. Patel, V. P., R. Barido, B. Johnson, and T. Ibarra, "Development of the Internal Thermal Control System (ITCS) for International Space Station (ISS)," SAE Paper 2001-01-2332, 31st International Conference on Environmental Systems, Orlando, Florida, July 2001.

2. Faust, M. B., S. J. Zhou, and I. M. Brubaker, "DualMembrane Gas Trap for the Space Station Freedom Internal Thermal Control System," SAE Paper 932162, 23rd International Conference on Environmental Systems, Colorado Springs, Colorado, July 1993.

3. Dittmer Supra, L., M. Faust, and S. Zhou, "Development of a Thermal Control System DualMembrane Gas Trap," SAE Paper 951461, 25th International Conference on Environmental Systems, San Diego, California, July 1995.

4. Humphrey, J., L. Supra, and M. Faust, "A Thermal Control System Dual Membrane Gas Trap for the International Space Station," SAE Paper 972410, 27th International Conference on Environmental Systems, Lake Tahoe, Nevada, July 1997.

5. Leimkuehler, T. O., C. Lukens, D. R. Reeves, and J. M. Holt, "Operational Experience with the Internal Thermal Control System Dual-Membrane Gas Trap," SAE Paper 2003-01-2565, 33rd International 
Conference on Environmental Systems, Vancouver, British Columbia, Canada, July 2003.

6. Leimkuehler, T. O., C. Lukens, D. R. Reeves, and J. M. Holt, "Recent Operational Experience with the Internal Thermal Control System Dual-Membrane Gas Trap," SAE Paper 2004-01-2428, 34th International Conference on Environmental Systems, Colorado Springs, Colorado, July 2004.

\section{ABBREVIATIONS AND ACRONYMS}

atm: atmosphere

ISS: International Space Station

ITCS: Internal Thermal Control System

LTL: Low Temperature Loop

min: minutes

MTL: Moderate Temperature Loop

N/A: Not applicable

PP: Polypropylene

PPA: Pump Package Assembly

psid: pounds per square inch differential

$\Delta \mathrm{P}$ : Pressure Drop 